\title{
Towards improved social distancing guidelines: Space and time dependence of virus transmission from speech-driven aerosol transport between two individuals
}

\author{
Fan Yang $\odot,{ }^{1}$ Amir A. Pahlavan $\odot,{ }^{1}$ Simon Mendez $\odot,{ }^{2}$ \\ Manouk Abkarian, ${ }^{3}$ and Howard A. Stone ${ }^{1}{ }^{1, *}$ \\ ${ }^{1}$ Department of Mechanical and Aerospace Engineering, Princeton University, Princeton, \\ New Jersey 08544 USA \\ ${ }^{2}$ Institut Montpelliérain Alexander Grothendieck, CNRS, University of Montpellier, 34095 Montpellier, France \\ ${ }^{3}$ Centre de Biochimie Structurale, CNRS UMR 5048-INSERM UMR 1054, University of Montpellier, \\ 34090 Montpellier, France
}

(Received 26 August 2020; accepted 20 October 2020; published 1 December 2020)

It is now recognized that aerosol transport contributes to the transmission of the SARS-CoV-2 virus. Here, we improve existing social distancing guidelines for airborne pathogens, which are typically given in terms of distance with vague statements about contact times. Also, estimates of inhalation of virus in a contaminated space usually assume a well-mixed environment, which is realistic for some, but not all, situations. In particular, we consider a local casual interaction of an infected individual and a susceptible individual, both maskless, account for the air flow and aerosol transport characteristics of speaking and breathing in a poorly ventilated space, and propose social distancing guidelines that involve both space and contact time, based on a conservative model of fluid dynamics of the interactions.

DOI: 10.1103/PhysRevFluids.5.122501

\section{INTRODUCTION}

Transmission of the virus SARS-CoV-2 during the presymptomatic and asymptomatic stages of the disease COVID-19 is estimated to be responsible for more than half of all of the cases $[1,2]$. The recognition that aerosols play a significant role in the transmission of SARS-CoV-2 raises the issue of quantifying the air exchange between asymptomatic individuals, who are sources of the virus, and healthy individuals, who are susceptible to infection. A common model for characterizing this situation is to evaluate the probability of infection based on the number $N$ of virions inhaled, relative to a characteristic dose $N_{\text {inf }}$ that, on average, produces infection; the corresponding probability of infection $p$ is then estimated as [3]

$$
p(N)=1-e^{-N / N_{\text {inf }}} .
$$

Past studies of the transmission of other viruses provide insights into the risk of infection by performing room-scale averages of the virion concentration, e.g., Refs. [4,5]. Recent studies applied to SARS-CoV-2 have similar features and allow an estimate of the number of inhaled virions by a susceptible individual in a space, e.g., a bathroom, airplane, laboratory, etc. [6-8]. Such well-mixed models are appropriate when the asymptomatic source individuals are well removed from healthy individuals, so that the timescale to mix in the environment is faster than the time for direct exchange of air between a source and a receiver.

\footnotetext{
*Author to whom correspondence should be addressed: hastone@ princeton.edu
} 
Here, we are concerned with casual conversations, where local air exchange between individuals is the dominant factor in determining the infection probability. In contrast with violent expiratory events, such as coughing and sneezing, which have been shown to be an important pathway in the transmission of pathogens from symptomatic individuals $[9,10]$, we focus on speaking and breathing, which are relevant to transmission during presymptomatic and asymptomatic stages. Some earlier studies have characterized qualitative features of the respiratory flow between two people using numerical simulations and experiments with mannequins [11,12] and a recent study has numerically probed spatial features of drop and aerosol transport between two people where one is an infected speaker [13]; see also the reviews [14-16].

We consider a poorly ventilated environment, in which the separation distance between an asymptomatic speaker and a healthy interlocutor is comparable to those recommended by the World Health Organization $(1 \mathrm{~m})$ and the Center for Disease Control (CDC) in the United States $(2 \mathrm{~m})$. Indeed, we can imagine that such local, air flow-driven virus exchange may occur in conversations at parties, across the table at lunch or in a conference room, on a train, etc. Recent studies provide estimates of the characteristic infection dose, $N_{\text {inf }} \approx 100-1000$, and, consistent with other modeling efforts, below we take the conservative value $N_{\text {inf }}=100[6,7,17]$. We assume the transport of aerosolized virions is that of a passive scalar, as justified below, and use a model of the time and space dependence of air flow in speech from our recent work [18]. Thus, to characterize the risk of infection we provide a space-time diagram of social distancing. These assessments, in the absence of mask wearing, should be combined with other global estimates to better understand the possible dynamics of pathogen exchange in different situations. These results, in our view, suggest always decreasing contact time to minimize spread of virus.

\section{TRANSPORT FEATURES OF SPEECH}

\section{A. Assumptions}

Given the complexity of the speech-driven aerosol transport, we make the following simplifying assumptions in our analysis. (1) Consistent with the estimates of the fluid dynamics below, and recently published experiments and numerical simulations [18], the expiratory flow from speech is a conical, quasisteady jet, with a horizontal extent set by the speaking time. (2) Both individuals are maskless and in a poorly ventilated environment, which we take as those spaces where the ambient flow speed is $O(1) \mathrm{cm} / \mathrm{s}$. (3) The infected individual speaks continuously and the susceptible individual only listens (and breathes). The disturbance imparted by the susceptible receiver on the oncoming jet is neglected; this assumption will be discussed in Sec. II B. (4) The ambient relative humidity $(\mathrm{RH})$, which is the ratio of partial pressure of water vapor to the equilibrium saturation vapor pressure of water, is not too large, so that droplets from speech will evaporate quickly and form aerosols. In Sec. II C we provide an example for $\mathrm{RH}=50 \%$.

We note that these assumptions yield a conservative estimate for the fluid dynamics for pathogen transmission in a local casual interaction. These assumptions also apply to a susceptible individual that joins a group and stands opposite an asymptomatic person who has been speaking for some time.

\section{B. Conical, quasisteady air flows characterize exhalation during speech}

The dynamics of the air flows in speaking and breathing are controlled by the Reynolds number, $\operatorname{Re}=2 a v_{0} / v$, where $a$ is a typical length of the opening of the mouth, $v_{0}$ is the average speed of the flow at the mouth (typical values are given in Tables I and II), and the kinematic viscosity of air $v \approx 1.5 \times 10^{-5} \mathrm{~m}^{2} / \mathrm{s}$. Thus, the Reynolds numbers are relatively high $\left(\operatorname{Re} \approx 10^{3}-10^{4}\right)$. Utilizing laboratory experiments of the air flow during speaking, numerical simulations of the Navier-Stokes equations for pressure-driven flows from an orifice, which mimics speech, and a mathematical model of these dynamics, our recent work [18] has shown that maskless speaking and breathing in poorly ventilated environments can produce a conical quasisteady, turbulent jet after a few seconds, 
TABLE I. Typical values related to speaking reported in the literature.

\begin{tabular}{lc}
\hline SARS-CoV-2 concentration in saliva $c_{v}$ & Average: $7 \times 10^{6} \mathrm{ml}^{-1} ;$ maximum: $2 \times 10^{9} \mathrm{ml}^{-1}[31]$ \\
\hline Total droplet production rate $j_{0}$ & $140-770 \mathrm{nl} / \mathrm{min}[35]$ \\
Flow velocity at the mouth $v_{0}$ & $1-5 \mathrm{~m} / \mathrm{s}[20,36-40]$ \\
Inhalation volume flux $Q_{r}$ & 0.1 liters $/ \mathrm{s}[6]$ \\
Half-cone angle $\alpha$ & $10^{\circ}-14^{\circ}[18]$ \\
\hline
\end{tabular}

as shown in Fig. 1. Sufficiently far away from the mouth, the unsteadiness of the inhaling/exhaling signal is barely visible and the jet behaves similar to a turbulent jet with constant flow rate. A typical laboratory measurement of the flow field produced by breathing is displayed in Fig. 1(a), while a simulation of the air flow produced by speaking is shown in Fig. 1(b). The typical cone half angle $\alpha$ is about $10^{\circ}-14^{\circ}$. Hence, the area of the cone will envelop the size of a human head already at $50 \mathrm{~cm}$ separation distance. To illustrate the magnitude of exhaled concentrations relevant to typical social distances, in the simulation shown in Fig. 1(b) we highlight a concentration contour $c=0.05$ (with $c=1$ at the mouth), which is comparable to the scale of the head a distance $1.6 \mathrm{~m}$ away (the right limit of the figure). Hence, non-negligible exhaled concentrations are found at distances comparable to social interactions.

We note that our experiments show that during speaking the effect of buoyancy at the scale of a few meters of jet motion [Fig. 1(a)] does not cause a substantial vertical motion beyond the size of a human head. Moreover, due to entrainment of the surrounding air, the thermal signature will decay inversely with distance, comparable to a passive scalar such as an aerosol, as discussed below, which further diminishes the buoyancy effects.

As illustrated in Fig. 1, for the typical Reynolds numbers $\operatorname{Re} \approx 10^{3}-10^{4}$, the expiratory flow is approximately jetlike and entrains surrounding air, whereas we expect the inhalatory flow to be approximately hemispherical, as sketched in Fig. 2; see Refs. [18,19]. As a consequence of the asymmetry of inhalation and exhalation, only a small portion of the inhaled breath comes from the exhaled material of the same individual. This may be verified easily by a simple model: If we assume the volume of air inhaled and exhaled in one breathing cycle is the same, denoted by $V$, then the radius $R$ of the inhaled hemisphere is $R=(3 V / 2 \pi)^{1 / 3}$. In the volume to inhale, only a small conical portion (the shaded region in Fig. 2) is occupied by the exhaled material, whose volume is $V_{e} \approx \pi\left[(R+a \cot \alpha)^{3} \tan ^{2} \alpha-a^{3} \cot \alpha\right] / 3$. Using $V=0.5$ liters [20] and $\alpha=12^{\circ}$, we have $V_{e} / V \approx 0.1$. Indeed, exploiting the series of numerical simulations of periodic breathing and speaking published in Ref. [18], which used an elliptic mouth with minor and major axes respectively 1 and $1.5 \mathrm{~cm}$, and a fixed cycle duration of $4 \mathrm{~s}$, then for very different flow-rate signals, and a volume per breath between 0.5 and 1.0 liters (with equal volumes exhaled and inhaled), we found that typically $10 \%$ of the exhaled material from one breath was inhaled in the next inspiration. Systematic experiments mimicking human breathing show that the reinhalation ratio is $2 \%-10 \%$ for $\operatorname{Re}=10^{3}-10^{4}[19]$. This means that when a person inhales, they mainly inhale the ambient air. It is thus the environment around the head of the (healthy) person that needs to be characterized to develop an estimate of the risk of pathogen uptake in these close encounters.

TABLE II. Parameters used in Figs. 3 and 4 for characterizing SARS-CoV-2 viral contamination by an asymptomatic individual speaking in a poorly ventilated space.

\begin{tabular}{lccccc}
\hline \hline$\phi_{0}$ & $c_{v}$ & $N_{\text {inf }}$ & $Q_{r}$ & $a$ & $\alpha$ \\
\hline $6 \times 10^{-9}, 1 \times 10^{-8}$ [only in Fig. 4(b)] & $7 \times 10^{6} \mathrm{ml}^{-1}$ & 100 & 0.1 liters $/ \mathrm{s}$ & $1 \mathrm{~cm}$ & $12^{\circ}$ \\
\hline \hline
\end{tabular}


(a)
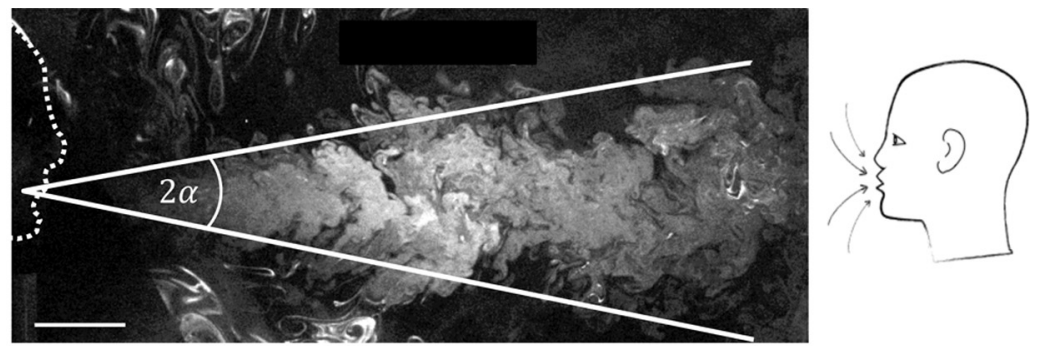

(b)

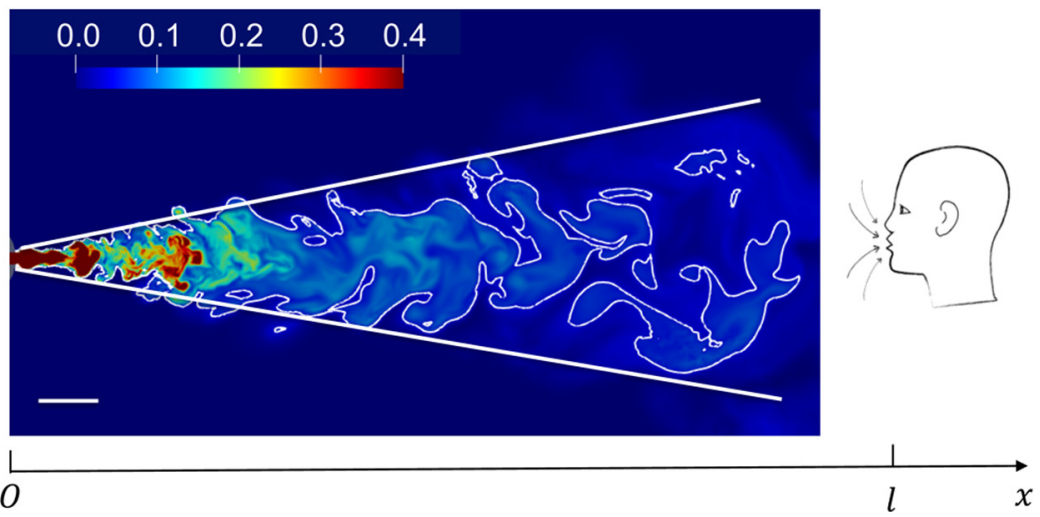

FIG. 1. Conical flows from breathing and speech. (a) Flow visualization of the conical jet produced by breathing from the mouth, adapted from Ref. [18]. Speaking can produce similar conical jets. The cone angle is $2 \alpha$. A (susceptible) receiver, shown with a head sketched on the right, is facing the asymptomatic (infected) breather/speaker at a distance $\ell$. (b) Simulated transport of exhaled material during repeated speech. Results of numerical simulations from Ref. [18]: Example of the instantaneous field of a passive scalar used to visualize the dilution of the air exhaled from an infected speaker, displayed over a vertical symmetry plane after 9.5 cycles of periodic speaking-inhaling (each cycle lasts $4 \mathrm{~s}$ ), with a volume per breath of 0.75 liters. The color map shows the concentration field $c$ of the passive scalar with $c=1.0$ at the exit of the mouth and $c=0$ in the ambient far field (the scale is saturated here to better visualize the field close to the receiver). An isoconcentration line at $c=0.05$ is displayed to help quantify the dilution levels far from the mouth of the speaker to the left. Both scale bars are $10 \mathrm{~cm}$.

\section{Evaporating drops and aerosols as passive tracers following the air flow}

Here, we compare the timescale of aerosol transport to that of evaporation and sedimentation. Experiments show that it takes $30-50 \mathrm{~s}$ for the jet to reach a distance $3 \mathrm{~m}$ for steady speaking [18]; the jet can reach $2 \mathrm{~m}$ in even $20 \mathrm{~s}$. The radii of droplets produced by speaking are found to be typically around or smaller than $5 \mu \mathrm{m}[6,21,22]$; drops of smaller sizes are traditionally categorized as aerosols. It takes about $0.1 \mathrm{~s}$ for a water droplet of radius $5 \mu \mathrm{m}$ (and about $2 \mathrm{~s}$ for radius $20 \mu \mathrm{m}$ ) to evaporate at $\mathrm{RH}=50 \%$ [23], so most small droplets produced by speaking should evaporate rapidly to become aerosol particles at the beginning of the jet spreading. We note that a recent numerical study shows that the the lifetime of droplets is significantly extended in a puff mimicking a strong cough, mainly due to the high local RH $(=100 \%)$ and the flow field in the puff [24].

During $\Delta t=50 \mathrm{~s}$, an aerosol particle of radius $r_{p}=1 \mu \mathrm{m}$ and density $\rho_{p}=10^{3} \mathrm{~kg} / \mathrm{m}^{3}$ sediments a distance of $h_{\text {sed }}=2 \rho_{p} g r_{p}^{2} \Delta t / 9 \mu_{a} \approx 5 \mathrm{~mm}$, where $g=9.8 \mathrm{~m}^{2} / \mathrm{s}$ is the gravitational acceleration and $\mu_{a}=1.8 \times 10^{-5} \mathrm{~Pa}$ s is the viscosity of air. Therefore, at the scale of a social interaction, we can ignore sedimentation of the small particles. Moreover, the Stokes number, $\mathrm{St}=\rho_{p} r_{p}^{2} v_{0} /\left(a \mu_{a}\right)$, which characterizes the tendency of the more dense particles to deviate from 


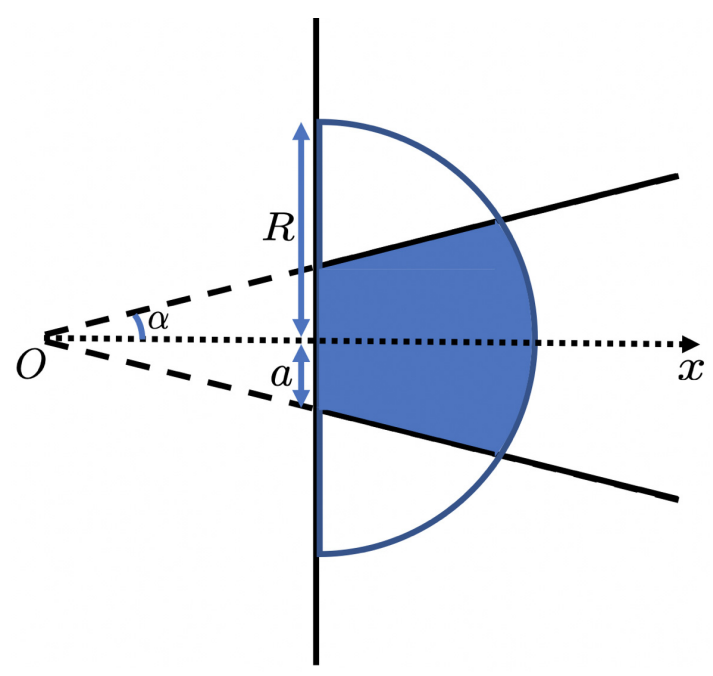

FIG. 2. Sketch of ideal respiratory flow regions. The expiratory flow region is a cone with half angle $\alpha$ and apparent origin at $O$. The mouth is modeled as a circle of radius $a$, located at $x=a \cot \alpha$. The inhalatory flow region is a hemisphere with radius $R$ (determined by the inhaled volume, see text) and centered at $x=a \cot \alpha$ (the exit of the mouth). In the inhaled volume, only the shaded region is from the previous exhalation.

the streamlines of the air flow, has a typical magnitude $\mathrm{St}=O\left(10^{-2}\right) \ll 1$. So, we treat the aerosol particles as passive tracers that follow the jet flow.

\section{AN ESTIMATE OF RISK FROM VIRUS INHALATION}

When an asymptomatic subject exhales or speaks, small droplets carrying virus can spread to a receiving interlocutor. To characterize the important aspects, we note that the drop size distribution [6,21,22,25-27], the influence of loudness and phonetic features on droplet production rates [28-30], and viral densities in saliva [31] have been reported in the literature. Here, we use these measurements, together with the flow field characteristics sketched in Sec. II B, to quantify the amount of virus that will reach the receiver in a poorly ventilated space, and so provide a measure of the risk of infection. The model allows quantitative assessment of the impact of separation distance and time of interaction.

The speaker's mouth can be approximated as a circle with radius $a$. Denoting the cone angle as $2 \alpha$ and the spatial axis from the cone vertex as $x$ (see Fig. 1), the cross-sectional area of the jet beyond the mouth is $A(x)=\pi(x \tan \alpha)^{2}$. Note that the radial size of the turbulent jet increases as it propagates due to entrainment of the surrounding air $[9,18,32]$. In a steady jet, with average velocity $v(x)$, the momentum flux, which is proportional to $v^{2}(x) A(x)$, is constant. Therefore $v(x)=v_{0} a / x \tan \alpha$, where $v_{0}$ is the flow velocity at the mouth. Denoting the virus concentration in the saliva of the asymptomatic speaker as $c_{v}$ (number virions/volume saliva) and the volume fraction of droplets at the mouth as $\phi_{0}$ (volume droplets/volume air), then the total droplet volume production rate in speaking is $j_{0}=\pi a^{2} v_{0} \phi_{0}$ (volume droplets/time), and the total emission rate of virus is $I_{0}=c_{v} j_{0}$ (number virions/time). Within a quasisteady approximation [18], and denoting $\phi(x)$ as the volume fraction of droplets in the jet, the flux of virus in the steady jet $c_{v} \phi(x) v(x) A(x)$ is also constant and equal to $I_{0}$. Thus, we conclude $\phi(x)=\phi_{0} a / x \tan \alpha$. Therefore, the speech-driven pathogen concentration $\left[c_{v} \phi(x)\right]$ decays as $x^{-1}$ with distance.

We now consider a susceptible individual at a distance $\ell$ in front of an infected speaker (Fig. 1). Assuming the average inhalation volume flux of the receiver (at $x=\ell$ ) to be $Q_{r}$, then the intake 

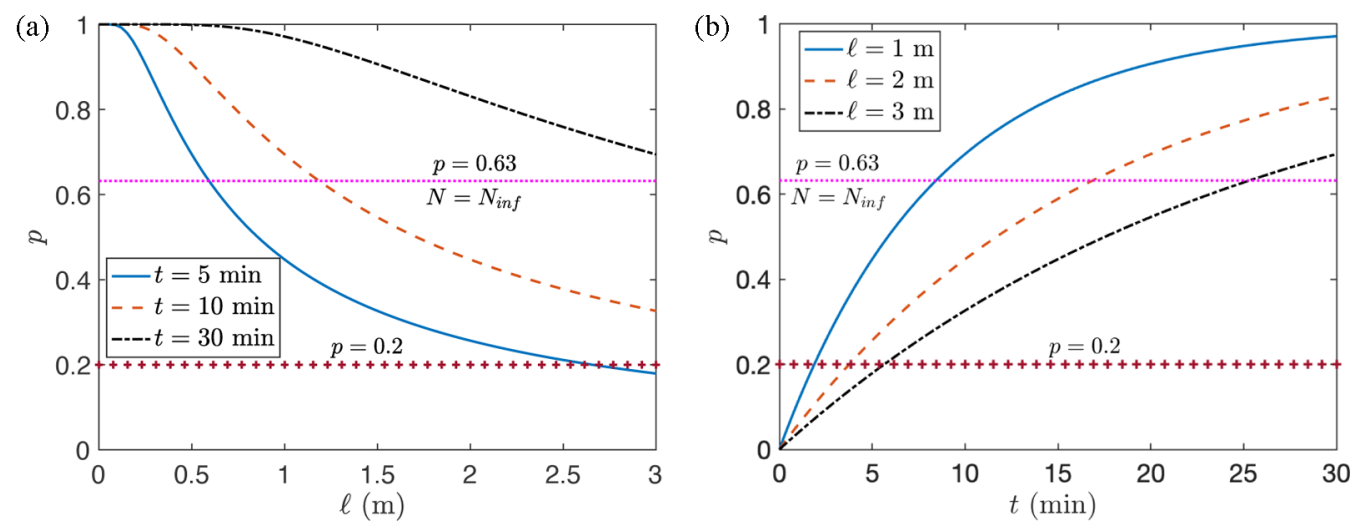

FIG. 3. Dependence of infection probability $p$ on (a) distance $\ell$ and (b) speaking time $t$ (for interaction with a single maskless asymptomatic speaker). Values of $\ell$ or $t$ for each curve are given in the legends. The spatial distance is truncated at $3 \mathrm{~m}$, beyond which we expect the ambient flows to become important even in a poorly ventilated space [18]. Two horizontal lines $p=0.63$ (or equivalently $N=N_{\text {inf }}$ ) and $p=0.2$ are plotted. Reported values in these figures are based on typical numbers listed in Table II, but large variations of $c_{v}$ are possible [34], with larger values implying a shorter contact time for the same risk of infection.

dose of the receiver over a period of time $t$ is

$$
N(\ell, t)=c_{v} \phi(\ell) Q_{r} t=\frac{c_{v} \phi_{0} a Q_{r} t}{\ell \tan \alpha} .
$$

This result does not depend explicitly on the speed of the air flow produced by the speaker. Also, we note that as the droplets evaporate $c_{v}$ will increase and $\phi(x)$ will decrease, however, the virus concentration $c_{v} \phi(x)$ (number virions/volume air) is unaffected by evaporation.

Typical values of the variables in Eq. (2) are summarized in Table I. The typical range of $\phi_{0}$ is calculated from Table I to be $2 \times 10^{-9}-1 \times 10^{-8}$. Note that the virus emission rate $I_{0}$ in breathing has been measured by collecting respiratory droplets and aerosols in $30 \mathrm{~min}$ from infected people [33]. The average value is around $300 \mathrm{~min}^{-1}$, which is smaller than $I_{0}$ in speaking: For an average infected patient $\left(c_{v}=7 \times 10^{6} \mathrm{ml}^{-1}, v_{0}=3 \mathrm{~m} / \mathrm{s}\right), I_{0} \approx 1 \times 10^{3}-5 \times 10^{3} \mathrm{~min}^{-1}$.

Although the typical "infectious dose" of SARS-CoV-2 is unknown, estimates have been provided. Consistent with other studies, we approximate $N_{\text {inf }}$ conservatively as $N_{\text {inf }}=100$ [6,7]. Based on this criterion and Eq. (2), we can plot the probability of infection $p$ versus distance $\ell$ and time $t$ as shown in Fig. 3. The results show the importance of distancing and decreasing contact time for reducing the probability of infection. In order to use a simple terminology, we define a boundary between lower- and higher-risk situations with the probability $p\left(N=N_{\text {inf }}\right)=1-e^{-1}=0.63$. From Fig. 3, a distance of $\ell=1 \mathrm{~m}$ can maintain a lower risk for an interaction of only $8 \mathrm{~min}$; for $\ell=2 \mathrm{~m}$, the interaction time should be restricted to less than $16 \mathrm{~min}$. If one wants to maintain the probability of infection under 0.2 , the corresponding contact times should be less than 2 and 4 min, respectively, as shown in Fig. 3. We remind the reader that these estimates are based on a single maskless infected individual in a poorly ventilated space (using average virus parameters reported in the literature). We reiterate that reported values in Fig. 3 are based on typical numbers, but large variations of $c_{v}$ are possible [34], with larger values implying a shorter contact time for the same risk of infection.

Next, we present a space-time, social distancing diagram of infection risk. In Fig. 4(a), we illustrate the probability of infection (yellow is higher and blue is lower), with spatial separation indicated on the horizontal axis and contact time on the vertical axis. This figure makes clear that at distances typically discussed for social distancing, longer contact times in speaking engagements with an asymptomatic individual increase the probability of infection. 

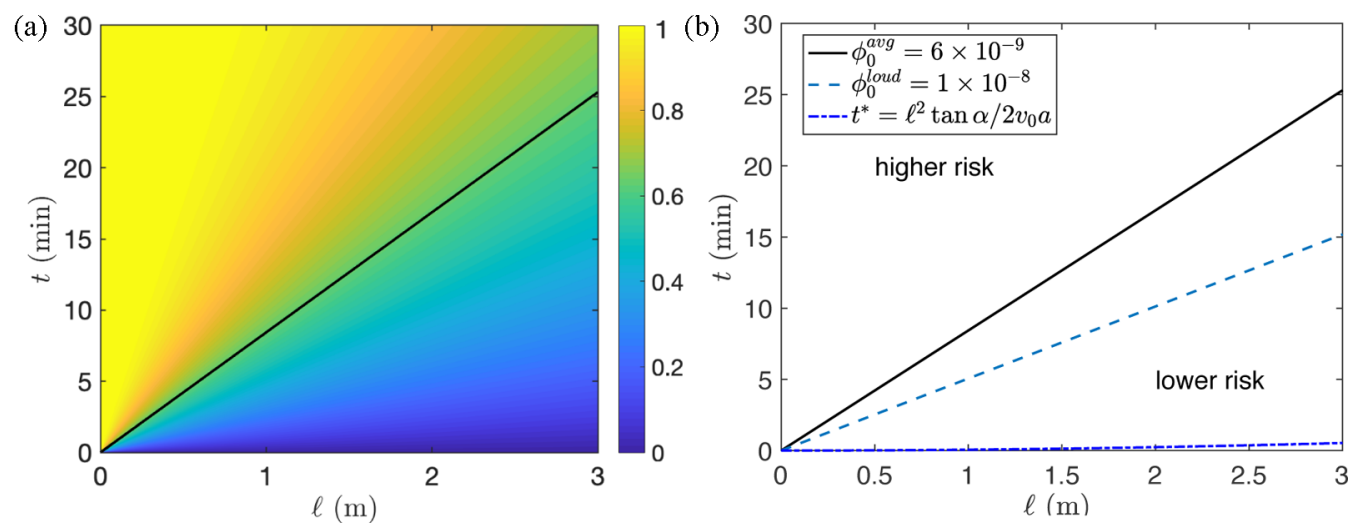

FIG. 4. Space-time diagram of infection risks. The color map (a) shows the infection risk $p$ as a function of distance $\ell$ and contact time $t$ with $\phi_{0}=6 \times 10^{-9}$, in which the straight line indicates $p=0.63$. (b) shows the effects of $\phi_{0}$. The infection risk is considered lower (higher) in the region below (above) the straight lines, where $p=0.63$ or, equivalently, $N=N_{\text {inf }}$ is proposed as a boundary for approximating relative risk. In (b) $t^{*}$ indicates the time for the jet to reach $\ell$ in the initial spreading stage. Below $t^{*}$ is the no-risk region. The plot is for a susceptible individual interacting with a single maskless asymptomatic speaker in a poorly ventilated space. Reported values in these figures are based on typical numbers listed in the legend and Table II, but large variations of $c_{v}$ are possible [34], with larger values implying shorter contact time for the same risk of infection.

Experiments show that the production rates of droplets increase with the loudness of speech [28-30], which is approximately examined in Fig. 4(b) by using $\phi_{0}^{\text {avg }}=6 \times 10^{-9}$ (typical speech) and $\phi_{0}^{\text {loud }}=1 \times 10^{-8}$ (loud speech). We observe that for $\ell=2 \mathrm{~m}$ and $\phi_{0}^{\text {avg }}$, and based on $N=N_{\text {inf }}$ as the estimate for increased risk, it is higher risk to speak with an asymptomatic individual for more than $16 \mathrm{~min}$, but with loud speech $\left(\phi_{0}^{\text {loud }}\right)$ for more than $10 \mathrm{~min}$. According to this model, when talking over $25 \mathrm{~min}$, there is a higher risk of infection even at a separation distance of $3 \mathrm{~m}$.

We note that for a speech-driven flow, the time for the jet to reach a distance $\ell$ is $t^{*}(\ell)=$ $\ell^{2} \tan \alpha / 2 v_{0} a$ [18]. In Fig. 4(b), the region below $t^{*}$ is therefore a "no-risk" region since there has not been time for any exhaled material to reach the receiver. Experiments show that $t^{*} \approx 30-50 \mathrm{~s}$ for the jet to reach $\ell=3 \mathrm{~m} \mathrm{[18],} \mathrm{which} \mathrm{matches} \mathrm{the} \mathrm{calculated} \mathrm{result} t^{*}=32 \mathrm{~s}$ using the parameters in Table II. The value of $t^{*}$ is usually much smaller than the critical time $t$ separating the higherand lower-risk regions in Fig. 4(b).

In all of above discussions an average virus concentration in saliva $c_{v}=7 \times 10^{6} \mathrm{ml}^{-1}$ is assumed. However, for a patient with a very high viral load in the saliva, $c_{v}$ can be as high as $2 \times 10^{9} \mathrm{ml}^{-1}$, which is 300 times larger than the average value [31]. Consequently, the time $t_{s}$ for $N\left(\ell, t_{s}\right)=N_{\text {inf }}$ at $\ell=3 \mathrm{~m}$ is $t_{s} \approx 5 \mathrm{~s}$ (using $\phi_{0}^{\text {avg }}=6 \times 10^{-9}$ and other parameters listed in Table II), which is much smaller than $t^{*}$ and means that the virion uptake by the receiver will surpass $N_{\text {inf }}$ almost as soon as the jet reaches them. Therefore, the infection probability for speaking with a patient with a very high viral load in the saliva (a potential superspreader) even for less than $1 \mathrm{~min}$ is high at a $3 \mathrm{~m}$ separation. Wearing masks can block the formation of jet, filter droplets, and aerosols, and thus mitigate against the transmission of airborne pathogens [41].

\section{CONCLUDING REMARKS}

We analyzed the spatial and temporal dependence of local virus transmission between a speaking asymptomatic individual and a susceptible listening individual in the absence of mask wearing and in a poorly ventilated environment. We used recent quantitative characteristics of speech [18] and typical viral loads of COVID-19 infected individuals. We show that both social distancing and decreasing contact time are important to keep the probability of infection low. Our analysis suggests 
that the mask-free social distancing guidelines, $1 \mathrm{~m}$ according to WHO and $2 \mathrm{~m}$ according to CDC in the United States, should be accompanied by contact-time guidelines. In particular, our estimates above, using typical values reported in the literature for droplet production rates and viral loads in saliva, suggest that in a poorly ventilated space, the probability of infection is relatively high for $\approx 8 \mathrm{~min}$ of contact time for $1 \mathrm{~m}$ separation distance and $\approx 16 \mathrm{~min}$ for $2 \mathrm{~m}$ of separation distance. If the infected speaker has a very high viral load in the saliva, the infection risk is high within less than $1 \mathrm{~min}$ for $\ell=3 \mathrm{~m}$ separation. A reader should recognize that the quantitative results in Figs. 3 and 4 are model dependent; in particular, variability in viral load and other features of the fluid dynamics can have a significant impact on the results.

Also, we emphasize that the model presented here is for a scenario when the infected individual is actively speaking and the susceptible individual is a passive listener, both maskless. Future research is required for a better understanding of the more complex flow between two (or more) speakers and its impact on aerosol transmission. Similar estimates and guidelines for the importance of contact time have been proposed recently for ventilated spaces, based on a well-mixed room model [42]. The important point to emphasize is that in social situations there are also time restrictions that should be imposed to lower the risk of infection.

\section{ACKNOWLEDGMENTS}

We thank the National Science Foundation for support via the RAPID Grant CBET 2029370 (Program Manager is Ron Joslin). We also thank the IRN "Physics of Living Systems" (CNRS/INSERM) for travel support for M.A.

[1] L. Ferretti, C. Wymant, M. Kendall1, L. Zhao, A. Nurtay, L. Abeler-Dörner, M. Parker, D. Bonsall, and C. Fraser, Quantifying SARS-CoV-2 transmission suggests epidemic control with digital contact tracing, Science 368, eabb6936 (2020).

[2] S. M. Moghadas, M. C. Fitzpatrick, P. Sah, A. Pandey, A. Shoukat, B. H. Singer, and A. P. Galvani, The implications of silent transmission for the control of COVID-19 outbreaks, Proc. Natl. Acad. Sci. USA 117, 17513 (2020).

[3] T. Watanabe, T. A. Bartrand, M. H. Weir, T. Omura, and C. N. Haas, Development of a dose-response model for SARS coronavirus, Risk Anal. 30, 1129 (2010).

[4] S. Rudnick and D. Milton, Risk of indoor airborne infection transmission estimated from carbon dioxide concentration, Indoor Air 13, 237 (2003).

[5] W. Yang, S. Elankumaran, and L. C. Marr, Concentrations and size distributions of airborne influenza A viruses measured indoors at a health centre, a day-care centre and on aeroplanes, J. R. Soc., Interface $\mathbf{8}$, 1176 (2011).

[6] D. Bonn, S. H. Smith, A. Somsen, C. van Rijn, S. Kooij, L. van der Hoek, and R. A. Bem, Probability of aerosol transmission of SARS-CoV-2, doi: 10.1101/2020.07.16.20155572.

[7] B. L. Augenbraun, Z. D. Lasner, D. Mitra, S. Prabhu, S. Raval, H. Sawaoka, and J. M. Doyle, Assessment and mitigation of aerosol airborne SARS-CoV-2 transmission in laboratory and office environments, J. Occup. Environ. Hyg. 17, 1129 (2020).

[8] J. M. Kolinski and T. M. Schneider, Superspreading events suggest aerosol transmission of SARS-CoV-2 by accumulation in enclosed spaces, arXiv:2007.14807.

[9] L. Bourouiba, E. Dehandschoewercker, and J. W. M. Bush, Violent expiratory events: On coughing and sneezing, J. Fluid Mech. 745, 537 (2014).

[10] P. Bahl, C. Doolan, C. de Silva, A. A. Chughtai, L. Bourouiba, and C. R. MacIntyre, Airborne or droplet precautions for health workers treating coronavirus disease 2019? J. Infect. Dis., (2020).

[11] P. V. Nielsen, I. Olmedo, M. R. de Adana, P. Grzelecki, and R. L. Jensen, Airborne cross-infection risk between two people standing in surroundings with a vertical temperature gradient, HVACR Res. 18, 552 (2012). 
[12] L. Liu, Y. Li, P. V. Nielsen, J. Wei, and R. L. Jensen, Short-range airborne transmission of expiratory droplets between two people, Indoor Air 27, 452 (2017).

[13] W. Chen, N. Zhang, J. Wei, H.-L. Yen, and Y. Li, Short-range airborne route dominates exposure of respiratory infection during close contact, Build. Environ. 176, 106859 (2020).

[14] R. Mittal, R. Ni, and J. H. Seo, The flow physics of COVID-19, J. Fluid Mech. 894, F2 (2020).

[15] R. Mittal, C. Meneveau, and W. Wu, A mathematical framework for estimating risk of airborne transmission of COVID-19 with application to face mask use and social distancing, Phys. Fluids 32, 101903 (2020).

[16] L. Bourouiba, The fluid dynamics of disease transmission, Annu. Rev. Fluid Mech. 53, 473 (2021).

[17] S. Basu, Computational characterization of inhaled droplet transport in the upper airway leading to SARSCoV-2 infection, doi: 10.1101/2020.07.27.20162362.

[18] M. Abkarian, S. Mendez, N. Xue, F. Yang, and H. A. Stone, Speech can produce jet-like transport relevant to asymptomatic spreading of virus, Proc. Natl. Acad. Sci. USA 117, 25237 (2020).

[19] F. R. Haselton and P. G. Sperandio, Convective exchange between the nose and the atmosphere, J. Appl. Phys. 64, 2575 (1988).

[20] J. Gupta, C.-H. Lin, and Q. Chen, Characterizing exhaled airflow from breathing and talking, Indoor Air 20, 31 (2010).

[21] C. Y. H. Chao, M. P. Wan, L. Morawska, G. R. Johnson, Z. D. Ristovski, M. Hargreaves, K. Mengersen, S. Corbett, Y. Li, X. Xie, and D. Katoshevski, Characterization of expiration air jets and droplet size distributions immediately at the mouth opening, J. Aerosol Sci. 40, 122 (2009).

[22] S. Shao, D. Zhou, R. He, J. Li, S. Zou, K. Mallery, S. Kumar, S. Yang, and J. Hong, Risk assessment of airborne transmission of COVID-19 by asymptomatic individuals under different practical settings, J. Aerosol Sci. 151, 105661 (2020).

[23] R. R. Netz, Mechanisms of airborne infection via evaporating and sedimenting droplets produced by speaking, J. Phys. Chem. B 124, 7093 (2020).

[24] K. L. Chong, C. S. Ng, N. Hori, R. Yang, R. Verzicco, and D. Lohse, Extended lifetime of respiratory droplets in a turbulent vapour puff and its implications on airborne disease transmission, arXiv:2008.01841.

[25] J. Duguid, The size and the duration of air-carriage of respiratory droplets and droplet-nuclei, Epidemiol. Infect. 44, 471 (1946).

[26] R. G. Loudon and R. M. Roberts, Droplet expulsion from the respiratory tract, Am. Rev. Respir. Dis. 95, 435 (1967).

[27] M. Abkarian and H. A. Stone, Stretching and break-up of saliva filaments during speech: A route for pathogen aerosolization and its potential mitigation, Phys. Rev. Fluids 5, 102301 (2020).

[28] S. Asadi, A. S. Wexler, C. D. Cappa, S. Barreda, N. M. Bouvier, and W. D. Ristenpart, Aerosol emission and superemission during human speech increase with voice loudness, Sci. Rep. 9, 2348 (2019).

[29] S. Asadi, A. S. Wexler, C. D. Cappa, S. Barreda, N. M. Bouvier, and W. D. Ristenpart, Effect of voicing and articulation manner on aerosol particle emission during human speech, PLoS One 15, e0227699 (2020).

[30] F. K. A. Gregson, N. A. Watson, C. M. Orton, A. E. Haddrell, L. P. McCarthy, T. J. R. Finnie, N. Gent, G. C. Donaldson, P. L. Shah, J. D. Calder, B. R. Bzdek, D. Costello, and J. P. Reid, Comparing the respirable aerosol concentrations and particle size distributions generated by singing, speaking and breathing, doi: 10.26434/chemrxiv.12789221.v1.

[31] R. Wölfel, V. M. Corman, W. Guggemos, M. Seilmaier, S. Zange, M. A. Müller, D. Niemeyer, T. C. Jones, P. Vollmar, C. Rothe, M. Hoelscher, T. Bleicker, S. Brünink, J. Schneider, R. Ehmann, K. Zwirglmaier, C. Drosten, and C. Wendtner, Virological assessment of hospitalized patients with COVID-2019, Nature (London) 581, 465 (2020).

[32] J. S. Turner, Buoyancy Effects in Fluids (Cambridge University Press, Cambridge, UK, 1979).

[33] N. H. L. Leung, D. K. W. Chu, E. Y. C. Shiu, K.-H. Chan, J. J. McDevitt, B. J. P. Hau, H.-L. Yen, Y. Li, D. K. M. Ip, J. S. M. Peiris, W.-H. Seto, G. M. Leung, D. K. Milton, and B. J. Cowling, Respiratory virus shedding in exhaled breath and efficacy of face masks, Nat. Med. 26, 676 (2020). 
[34] T. C. Jones, B. Mühlemann, T. Veith, G. Biele, M. Zuchowski, J. Hoffmann, A. Stein, A. Edelmann, V. M. Corman, and C. Drosten, An analysis of SARS-CoV-2 viral load by patient age, doi: 10.1101/2020.06.08.20125484.

[35] V. Stadnytskyi, C. E. Bax, A. Bax, and P. Anfinrud, The airborne lifetime of small speech droplets and their potential importance in SARS-CoV-2 transmission, Proc. Natl. Acad. Sci. USA 117, 11875 (2020).

[36] Y. Chi, K. Honda, J. Wei, H. Feng, and J. Dang, Measuring oral and nasal airflow in production of Chinese plosive, in Proceedings of INTERSPEECH 2015, edited by S. Moller, H. Ney, B. Möbius, E. Nöth, S. Steidl (International Speech Communication Association, Dresden, Germany, 2015), pp. 2167-2171.

[37] J. D. Subtelny, J. H. Worth, and M. Sakuda, Intraoral pressure and rate of flow during speech, J. Speech Hear. Res. 9, 498 (1966).

[38] N. Isshiki and R. Ringel, Air flow during the production of selected consonants, J. Speech Hear. Res. 7, 233 (1964).

[39] J. Machida, Air flow rate and articulatory movement during speech, Cleft Palate J. 4, 240 (1967).

[40] C. H. Shadle, The aerodynamics of speech, in The Oxford Handbook of Innovation, edited by W. J. Hardcastle, J. Laver, and F. E. Gibbon (Wiley-Blackwell, Hoboken, NJ, 2010), Chap. 2, pp. 39-80.

[41] D. K. Chu, E. A. Akl, S. Duda, K. Solo, S. Yaacoub, and H. J. Schünemann, Physical distancing, face masks, and eye protection to prevent person-to-person transmission of SARS-CoV-2 and COVID-19: A systematic review and meta-analysis, Lancet 395, 1973 (2020).

[42] M. Z. Bazant and J. W. M. Bush, Beyond six feet: A guideline to limit indoor airborne transmission of COVID-19, doi: 10.1101/2020.08.26.20182824. 